\title{
Stability Control for Four-wheel Independent Drive Electric Vehicle Based on Variable Universe Fuzzy Control
}

\author{
Jun Wang, Zi Yang, Jinrui Nan, Mingxing Qiao \\ Beijing Institute of Technology, Collaborative Innovation Center of Electric Vehicles in Beijing, Beijing, \\ 100081, China
}

\begin{abstract}
For fully taking the advantage of independently controllable of torque of Four-wheel Independent Drive Electric Vehicle to enhance its stability, a 2-DOF vehicle reference model is set up based on Vehicle Dynamics foundation. On this basis, a variable universe fuzzy control strategy witch chose yaw rate as the control variable is designed. Thus, for four-wheel motor torque adjustments are applied to adjust the yaw moment of the vehicle and achieved its stability control target. The variable universe fuzzy control method is verified by using Matlab/Simulink and CARSIM co-simulation environment. The results show that the variable universe fuzzy control strategy can keep yaw rate and sideslip angle in the range of stable level and enhance handling stability of vehicle.
\end{abstract}

KEYWORD: Four-wheel Independent Drive Electric Vehicle; Variable Universe Fuzzy Control; Stability control

\section{INTRODUCTION}

For four-wheel independent drive electric vehicle, the torque of each wheel can controlled independently.it has a great advantage compared with traditional central driven vehicle. In addition, the motor has fast response speed, and its rotate speed or torque can easily measure. In the practical application, usually control the vehicle yaw moment to enhance the vehicle stability by adjusting the fourwheel drive torque.

For stability control of four-wheel independent drive electric vehicle, many control strategies are proposed in literature. In paper (Zhai L, 2010), an electronic differential speed controller for fourwheel independent drive vehicle is presented. Its controller uses neural networks PID theory to coordinately distribute the torque of four motors and enhance the steering stability of vehicle at a low speed. Several studies designed sliding mode variable structure controller with yaw rate, mass-center sideslip angle and both yaw rate and mass-center sideslip angle as control variables respectively, The controller can effectively enhance the handling stability of vehicle in extreme working conditions(Lin C, 2015). Paper (Song J, 2012) chose yaw rate and sideslip angle as the joint control variables to design the yaw moment control strategy, and used the theory of fuzzy control with the vehicle speed and road adhesion coefficient as the input to determine the joint control parameters and adjust the four-wheel drive torque.
Due to the fuzzy control does not depend on the control object, has a strong adaptive ability and good control effect to the nonlinear, large delay and other complex system, it has been widely applied in automobile yaw moment control. Over the past decade, the variable universe fuzzy control (VUFC) has developed based on the theory of fuzzy control. It is, in fact, by choosing the appropriate contractionexpansion factor to change the variable universes as the error changes. So it makes the actual fuzzy control rules dramatically increased. The closer to the expect control points, the smaller its control grades. $\mathrm{T}$ hus its can effectively get rid of disadvantages of limit control rules of general fuzzy controller (Gao $\mathrm{S}$ Z, 2010).

In this paper, a yaw moment control strategy for four-wheel independent drive electric vehicle is proposed based on the variable universe fuzzy control theory. It can control the four-wheel motors respectively, adjust their drive torque to correct the yaw moment to a desired value to enhance vehicle stability. The simulation is conducted in CARSIM and Matlab/Simulink co-simulation environment, and the results show the yaw rate and sideslip angle of the EV can be controlled to track the desired value, it show the validity of variable universe fuzzy control strategy used in EV stability control. 
2 VEHICLE 2-DOF DYNAMICS MODEL

At the beginning of the research, In order to study the vehicle plane motion and analyze the vehicle handling stability, a vehicle dynamics model should be constructed. In this paper, the controller is based on a 2-DOF dynamics model, it can reflect the vehicle driving state and the Intentions of the driver. In the analysis, (1) the front wheel steering angle is a direct input; (2) Ignore the influence of the nonlinear factors of steering system and the effect of vehicle suspension; (3) Ignore the air resistance and rolling resistance; (4) The vehicle speed along the $\mathrm{x}$ axis is constant and the tire cornering characteristics is considered in a linear range; (5) The roll angle of the $\mathrm{x}$ axis, the pitch angle of the $y$ axis and the position along the $\mathrm{z}$ axis are always considered zero. Therefore, the vehicle only has the lateral movement along the $\mathrm{y}$ axis and the yaw motion around the $\mathrm{z}$ axis. As shown in figure 2.1 , the vehicle will be simplified to 2-DOF dynamics model.

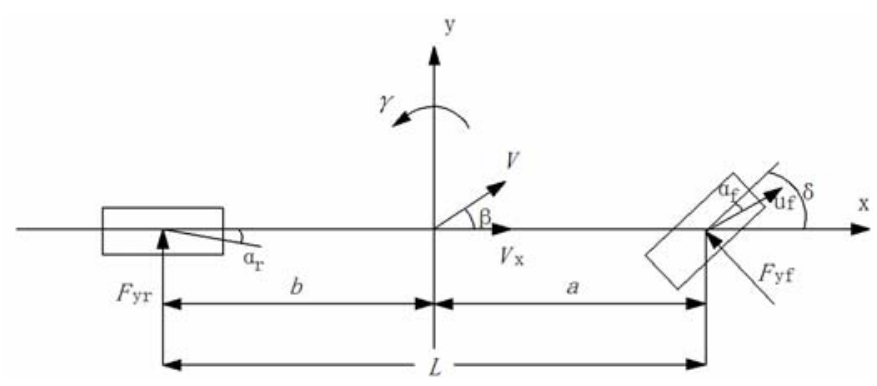

Fig.2.1 Vehicle 2-DOF Dynamics Model

The Steady state response of 2-DOF vehicle model can well reflect the steady driving state of the vehicle, it can be a reference dynamics model to obtain the desired state value. The Kinematic equations of the model can be described as:

$$
\begin{aligned}
& m u(\dot{\beta}+\gamma)=\left(C_{f}+C_{r}\right) \beta+\frac{1}{u}\left(a C_{f}-b C_{r}\right) \gamma-C_{f} \delta \\
& I_{z} \gamma=\left(a C_{f}-b C_{r}\right) \beta+\frac{\left(a^{2} C_{f}+b^{2} C_{r}\right)}{u} \gamma-a C_{f} \delta
\end{aligned}
$$

And it can be described in Equation of state matrix form:

$$
\left[\begin{array}{c}
\dot{\beta} \\
\dot{\gamma}
\end{array}\right]=\left[\begin{array}{cc}
\frac{C_{f}+C_{r}}{m v_{x}} & -1+\frac{a C_{f}-b C_{r}}{m v_{x}^{2}} \\
\frac{a C_{f}-b C_{r}}{I_{z}} & \frac{a^{2} C_{f}+b^{2} C_{r}}{I_{z} v_{x}}
\end{array}\right]\left[\begin{array}{l}
\beta \\
\gamma
\end{array}\right]+\left[\begin{array}{c}
-\frac{C_{f}}{m v_{x}} \\
-\frac{a C_{f}}{I_{z}}
\end{array}\right] \delta
$$

Where $\mathrm{m}$ is the total mass of the vehicle, $\beta$ is mass-center side-slip angle; $I_{z}$ is yaw rotational inertia; $\gamma$ is the yaw rate; $v$ is the velocity of vehicle. $C_{f}, C_{r}$ are the front wheel cornering stiffness and rear wheel cornering stiffness; $a$ is the distance from centroid to front axle, $b$ is the distance from centroid to rear axle. $\delta$ is the front wheel angle. $F_{y f}, F_{y r}$ are the lateral force of the front wheel and rear wheel. $\alpha_{f}$ is the front wheel side slip angle and $\alpha_{r}$ is the rear wheel side slip angle.

We choose the steady steering performance of the 2-DOF vehicle model as a characterization of vehicle steering stability, thus the yaw rate of the 2-DOF vehicle model is considered as a desired yaw rate. When. the vehicle reaches a steady state, $\beta=0, \gamma=0$. The steady-state value of the yaw rate of the 2-DOF vehicle reference model can be obtained from equation(3):

$\gamma_{d}=\frac{v_{x}}{1+K v_{x}} \frac{\delta}{L}$

Where:

$K=\frac{m}{L^{2}}\left(\frac{a}{C_{r}}-\frac{b}{C_{f}}\right)$ is vehicle stability factor.

The yaw rate is limited by the capacity of the road adhesion:

$\left|\gamma_{\max }\right|=\frac{\mu g}{v_{x}}$

Where $\mu$ is Road adhesion coefficient.

\section{YAW MOMENT CONTROL SYSTEM DESIGN}

\subsection{Structure of control system}

The principle of control system is shown in figure 3.1.Vehicle velocity and steering wheel angle signal is inputted into the 2-DOF vehicle reference model. The desired yaw rate is calculated and outputted from the reference model. Yaw moment controller then calculate the additional yaw moment needed to keep the vehicle stability based on the error between the actual vehicle yaw rate and desired yaw rate. In the end, the system allocate the yaw moment obtained from the controller to four wheel motors to achieve the control effect.

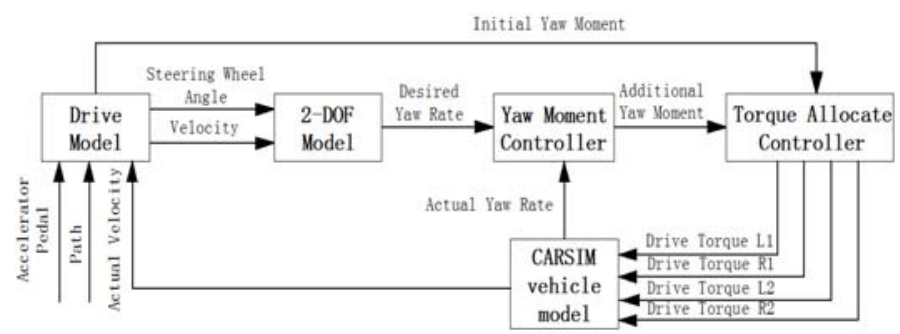

Fig.3.1 structure of control system

\section{2 variable universe fuzzy controller design}

General fuzzy controller can not modify its structure once determined. Its input and output variables relative to their domain is too large or too small because of Lack of adaptive capacity. Thus it is Difficult to guarantee to achieve the desired control accuracy. Li H X First proposed fuzzy variable universe adaptive fuzzy control (Li H X, 2002). Under the condi- 
tions of constant rules, it can makes fuzzy universe contract when the error becomes smaller or expand when the error becomes larger. It is equivalent to increase the control rules so that it can improve the control accuracy.

Set the initial input fuzzy universe as:

$X=[-E, E], Y=[-U, U]$

Where:

$$
\begin{aligned}
& X\left(x_{i}\right)=\left[-\alpha_{i}\left(x_{i}\right) E, \alpha_{i}\left(x_{i}\right) E\right] \\
& Y\left(y_{j}\right)=\left[-\beta_{j}\left(y_{j}\right) E, \beta_{j}\left(y_{j}\right) E\right]
\end{aligned}
$$

Where:

$$
\alpha_{i}\left(x_{i}\right)(i=1,2, \cdots, n) \text { and } \beta_{j}\left(y_{j}\right)(j=1,2, \cdots, m) \text { are }
$$

contraction-expansion factors of the universe $X$ and $Y$.The Schematic diagram of the contraction and expansion of fuzzy universe is shown in figure 3.2 .
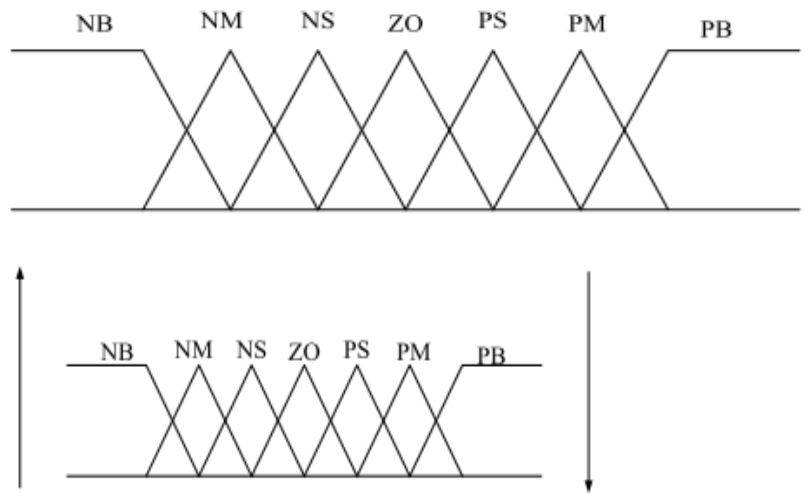

Fig.3.2 contraction and expansion of fuzzy universe

In this paper, a variable universe fuzzy control logic is proposed based on general fuzzy control theory .The structure of controller is shown in figure 3.3.

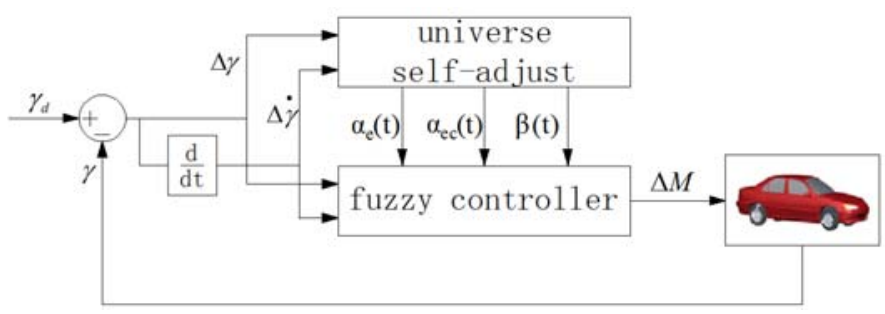

Fig.3.3 structure of variable universe fuzzy controller

Wherein the difference $\Delta \gamma$ and the rate of its change $\Delta \gamma$ between the actual yaw rate and the desired yaw rate are the input variables. The additional yaw moment $\Delta M$ is the output variable. The fuzzy subset of $\Delta \gamma, \Delta \gamma$ and $\Delta M$ are all $\{\mathrm{NB}$, NM,NS, O,PS,PM,PB). Where the initial universe: $\Delta \gamma$ : $[-E, E], \Delta \gamma:[-E C, E C], \Delta M:[-U, U]$ are all($6,6)$.The membership function is shown in figure 3.4 .

To determine the function of contractionexpansion factors is a key part of the design of vari- able universe fuzzy controller. Factor $\alpha_{i}\left(x_{i}\right)$ and $\beta_{j}\left(y_{j}\right)$ must satisfies the following axioms: (1) duality; (2) Nearzero; (3) monotonicity; (4) compatibility; (5) normality. In this paper, the function of contraction-expansion factors is as in Eq. (6)(7)(8):

$$
\begin{aligned}
& \alpha(e)=1-\lambda \exp \left(-k e^{2}\right), \lambda \in(0,1), k>0 \\
& \alpha(e c)=1-\lambda \exp \left(-k e c^{2}\right), \lambda \in(0,1), k>0 \\
& \beta(u)=(|u| / U)^{\tau}+\varepsilon, \tau>0
\end{aligned}
$$

Where $e$ and $e c$ are inputs, $u$ is output, $\varepsilon$ is a very small positive constant. Here choose $\lambda=0.99$, $k=0.5, \tau=0.85$.

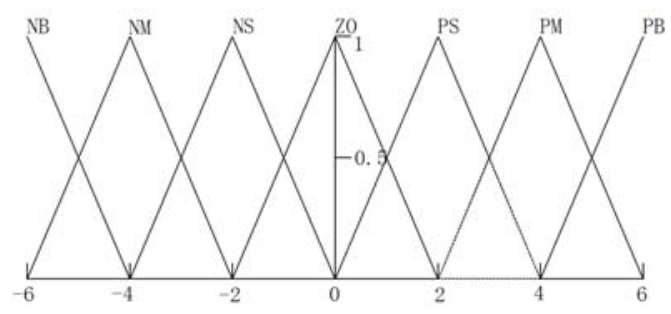

Fig.3.4 Input and output membership functions

\section{SIMULATION RESULTS}

In this section, the variable universe fuzzy controller was simulated in Matlab/Simulink and CARSIM cosimulation environment to verify the feasibility of yaw moment control system .The main parameters used in the simulation model are shown as in table4.1.

Table4.1Parameters In Simulation Model

\begin{tabular}{cc}
\hline Parameters & Value \\
\hline Vehicle Mass (kg) & 1411 \\
Long/Width/Height (m) & $4050 / 1600 / 1846$ \\
Distance From C. G. To Front Axle (m) & 1.04 \\
Distance From C. G. To Rear Axle (m) & 1.56 \\
Axle Track (Front/Rear) (m) & 1.48 \\
Wheel Effective Radius & 0.3 \\
Height of C. G. (m) & 0.54 \\
Vehicle Yaw Inertia (kg • m2) & 2031.4 \\
\hline
\end{tabular}

A sever Double lane change manoeuver (DLC) was simulated. The initial velocity is $90 \mathrm{Km} / \mathrm{h}$. The road friction coefficient is 0.4 . With variable universe fuzzy control and without control simulation is applied respectively, the results is shown in figure 4.1-4.4. 


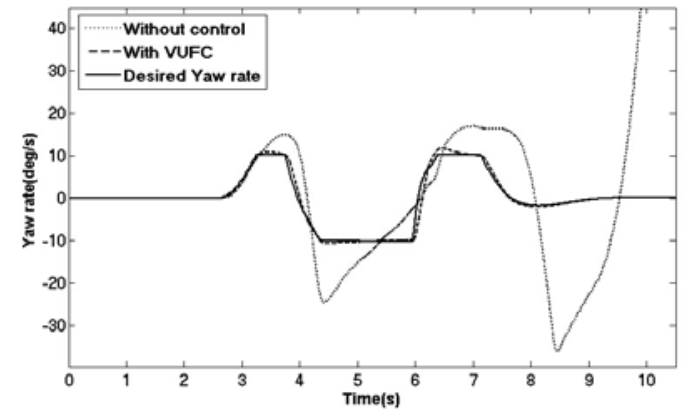

Fig.4.1 Yaw rate responses

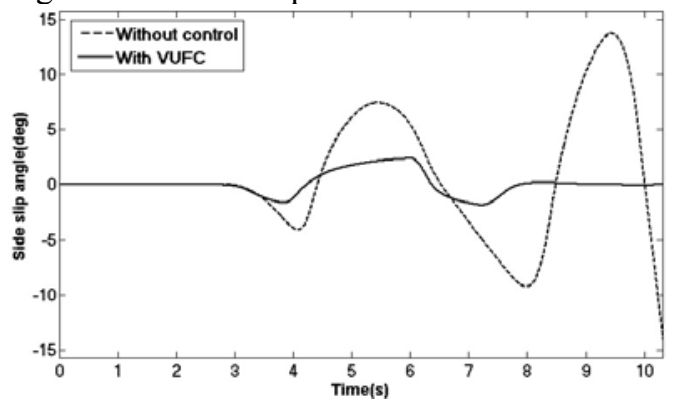

Fig.4.2 Sideslip angle responses

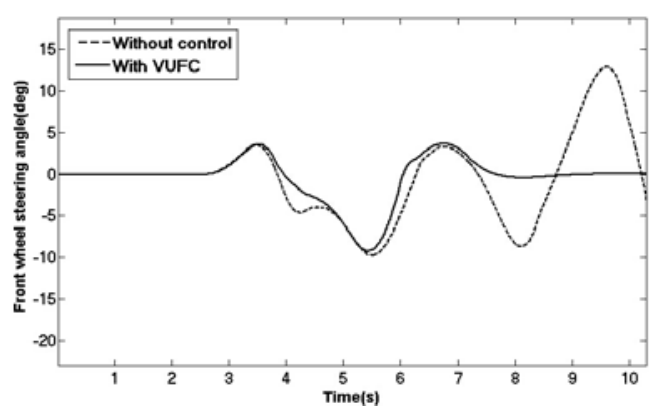

Fig.4.3 Front wheel steering angle

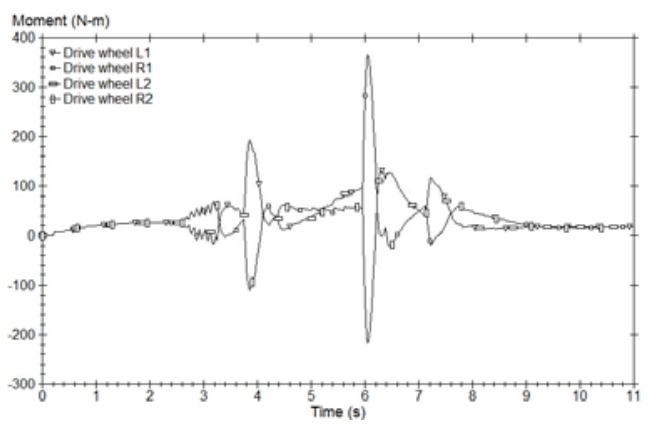

Fig.4.4 Driving torque responses

The results shown in figure 4.1 and 4.1 verified with the VUFC method proposed in this paper, the sideslip angle is restrained and the yaw rate is kept well. Without the control, the sideslip angle and the yaw rate have very dramatic change. It means the vehicle lost handling stability. In figure 4, the front wheel steering angle had a significant fluctuation and the driver can not control the vehicle well, while the value kept steady with the VUFC method. The result of driving torque allocate is shown in figure 4.4 .

\section{CONCLUSIONS}

A variable universe fuzzy control method is proposed for the four-wheel independent drive electric vehicle. The research results show:

1) The variable universe fuzzy controller can dynamically adjust its universe with the change of input variables.

2) With the yaw moment control system, the yaw rate and sideslip angle follow its target accurately and smoothly. Simulation results show that the steering maneuvering and stability of vehicle can be improved.

\section{REFERENCES}

Gao S Z,Gao X W,Zhu Z C.Stripper Temperature Control Method Based on Variable Universe Fuzzy-PID.Journal of Northeastern University(Natural Science).2010,31

(10):1369-1372.

Lin C,Peng C L,Cao W K.Sliding Mode Varia ble Structure Control for the Stability of Independent Drive Electric Vehicle.Automotive Engineering,2015,37(2):132-138.

Massimo Canale,Lorenzo Fagiano,AntonellaFerrara,e-t al.Vehicle Yaw Control via Second-Order Sliding-Mode Technique.IEEE Transactions on Industrial Electronics, 2008,55(11):3908-3916.

Li F Q,Wang J,Liu Z D.Fuzzy-logic-based Controller Design for Four-wheel-drive Electric Vehicle Yaw Stability Enhancement.Proceedings of the 2009 Sixth International Conference on Fuzzy Systems and Knowledge Discovery,FSKD 2009:116-120.

Li G,Wang C,Shi J,Han H L.Direct Yaw Moment Control Based on Fuzzy Control.Computer Simulation.2014,31 (12):151-155.

WANG Q,WANG Q,CHEN W.Advanced variable universe fuzzy control of with magneto-rheological Suspension.Journal of Vibration Engineering. 2009, 22

(5):512-518.

Li H X,Miao Z H,Wang J Y Variable universe ada-ptive fuzzy control on the quadruple inverted pendul-um.Science in China Series E-Technological Sciences,2002,45(2):21 3224.

Li H X. Adaptive fuzzy controllers based on variable universe.Science in China Series E-Technological Sciences, 1999,42(1):10-20.

Song J. Integrated Control Of Brake Pressure And Rear-wheel Steering To Improve Latereal Stability With Fuzzy Logic. International Journal of Automotive Technology,2012,13(4): 563-570.

Zhai L, Dong S Q, Luo K Y.Electronic Differential Speed Steering Control for Four In-Wheel Motors Independent Drive Vehicle. 2010,30(8) :901-905.

Zhao Y E,Zhang J W.Stability Control for a Four-MotorWheel Drive Electric Vehicle Based on Sliding Mode Control.JOURNAL OF SHANGHAI JIAOTONG UNIVERSITY,2009,43(10):1526-1530. 